\title{
FINITE GROUPS WITH SOLVABLE MAXIMAL SUBGROUPS
}

\author{
J. W. RANDOLPH
}

R. Baer [1] has established relationships between maximal subgroups and minimal normal subgroups. We note here some applications of these relationships if a group has a solvable maximal subgroup.

All groups considered are finite. If $H$ is a subgroup of a group $G$, we denote the centralizer of $H$ in $G$, the normalizer of $H$ in $G$ and the largest normal subgroup of $G$ in $H$ by $C(H), N(H)$ and core $(H)$, respectively. The identity of $G$ will be denoted by 1 , and the order of $G$ by $|G|$.

THEOREM. Let $G$ be a group containing a solvable maximal subgroup $S$ and a non-Abelian minimal normal subgroup $M$. Then

(1) $C(M)$ and core $(S)$ are both equal to the intersection of all solvable maximal subgroups of $G$,

(2) $S$ is not normal in $G$,

(3) $M$ is the only non-Abelian minimal normal subgroup of $G$,

(4) $\operatorname{core}(S)=1$ if and only if $M$ is the only minimal normal subgroup of $G$,

(5) if $S$ is nilpotent then the intersection of all solvable maximal subgroups of $G$ is equal to the intersection of all nilpotent maximal subgroups of $G$, the 2-Sylow subgroup $P$ of $S$ is a non-Abelian 2-Sylow subgroup of $G$, and $N(P)=S$, and

(6) if $S$ is nilpotent and $\operatorname{core}(S)=1$ then $S$ is a non-Abelian 2-Sylow subgroup of $G$.

Proof.

Lemma [1, pp. 119-120]. Let $G$ be a group containing a maximal subgroup $S$ such that $\operatorname{core}(S)=1$. Then

(a) if $H$ is a normal subgroup of $G$ and $H \neq 1$, then $C(H) \cap S=1$ and $C(H)=1$ or $C(H)$ is a minimal normal subgroup of $G$,

(b) there exist at most two different minimal normal subgroups of $G$, and

(c) if $A$ and $B$ are two different minimal normal subgroups of $G$, then $A, B$ and $A B \cap S$ are isomorphic.

Received by the editors April 28, 1969. 
Let $G^{*}=G / \operatorname{core}(S), S^{*}=S / \operatorname{core}(S)$ and $M^{*}=M \operatorname{core}(S) / \operatorname{core}(S)$. Since solvable minimal normal subgroups of finite groups are Abelian [3, p. 74], $M \nsubseteq S$, so $M^{*}$ is a minimal normal subgroup of $G^{*}$. Also $\operatorname{core}\left(S^{*}\right)=1^{*}$, the identity of $G^{*}$, as noted by Baer $[1$, p. 123]. Moreover $M \cap \operatorname{core}(S)=1$, whence $M$ and $M^{*}$ are isomorphic and core $(S)$ $\subseteq C(M)$. If $M^{*}$ and $N^{*}$ were distinct minimal normal subgroups of $G^{*}$, then by the lemma, $M^{*}$ and $M^{*} N^{*} \cap S^{*}$ would be isomorphic; but $S$ is solvable so then $M^{*}$ (and hence also $M$ ) would be solvable, a contradiction. So $M^{*}$ is the only minimal normal subgroup of $G^{*}$. If $C\left(M^{*}\right) \neq 1^{*}$, then by the lemma $C\left(M^{*}\right)$ is a minimal normal subgroup of $G^{*}$, so $C\left(M^{*}\right)=M^{*}$ and $M^{*}$ is Abelian, a contradiction. Thus $C\left(M^{*}\right)=1^{*}$. But $C\left(M^{*}\right)=C(M) \operatorname{core}(S) / \operatorname{core}(S)$, and consequently $C(M) \subseteq \operatorname{core}(S) \subseteq S$. Let $R$ denote the intersection of all solvable maximal subgroups of $G$. Then $C(M) \subseteq R$. But $R$ is a solvable normal subgroup of $G$, so $M \cap R=1$ and $R \subseteq C(M)$, proving (1).

If $S$ were normal in $G, G^{*}$ would have prime order and $M^{*}$ (and hence also $M$ ) would be Abelian, a contradiction, proving (2).

If $G$ had a non-Abelian minimal normal subgroup $N$ such that $N \neq M$, then by (1), $C(M)=C(N)$. But since $M$ and $N$ are both minimal normal and $M \neq N, M \cap N=1$, so $M \subseteq C(N)=C(M)$ and $M$ is Abelian, a contradiction, and (3) is proved. The truth of (4) is evident.

For the remainder of the proof, suppose $S$ is nilpotent. The first assertion of (5) follows from proving in the same manner as for (1) that $C(M)$ is the intersection of all nilpotent maximal subgroups of $G$. If a group $G$ has a nilpotent maximal subgroup whose 2-Sylow subgroup is Abelian, then $G$ is solvable [2, p. 281]. But $M$ is not Abelian, so the 2-Sylow subgroup $P$ of $S$ is not Abelian.

Suppose additionally that core $(S)=1$. Then $S$ must be a Hall subgroup of $G$, since otherwise [3, p. 392] $G$ would have an Abelian minimal normal subgroup, contradicting (4) of our theorem. Assume $S$ is not a Sylow subgroup of $G$. Let $Q$ be any Sylow subgroup of $S$ with $Q \neq 1$. Since $S$ is nilpotent, $Q$ is normal in $S$. Thus $S \subseteq N(Q)$, and by the maximality of $S, S=N(Q)$ or $Q$ is normal in $G$. But core $(S)=1$ so $S=N(Q)$. Therefore [3, p. 377] $S$ has a normal complement, say $K$, in $G$. Since $S$ is nilpotent, $G / K$ must be solvable. But $S$ is a Hall subgroup of $G$ and $S$ has even order, so $K$ has odd order, whence $K$ is solvable, by the Feit-Thompson theorem. But then $G$ is solvable, a contradiction, proving (6).

If $\operatorname{core}(S) \neq 1$, consider $S^{*}$ which is a nilpotent maximal subgroup of $G^{*}$ and satisfies core $\left(S^{*}\right)=1^{*}$. Applying (6) to $S^{*}$, we see that $S^{*}$ is a non-Abelian 2-Sylow subgroup of $G^{*}$. Hence $\left[3\right.$, p. 134] $S^{*}$ 
$=J \operatorname{core}(S) / \operatorname{core}(S)$ for some non-Abelian 2-Sylow subgroup $J$ of $G$. Then $\left|S^{*}\right|=|J / J \cap \operatorname{core}(S)|$. But $|S|=\left|S^{*}\right||\operatorname{core}(S)|$, so $|S|$ $=|J||\operatorname{core}(S)| /|J \cap \operatorname{core}(S)|$. It follows that $P$, the 2 -Sylow subgroup of $S$, is a non-Abelian 2-Sylow subgroup of $G$. Finally, as before, $S=N(P)$ or $P$ is normal in $G$; in the latter case $P=J, J \subseteq$ core $(S)$ and $S^{*}$ is Abelian, a contradiction. This completes the proof of (5) and the theorem.

REMARK. It follows that if a non-Abelian simple group $G$ has a nilpotent maximal subgroup $S$, then $S$ is a non-Abelian 2-Sylow subgroup of $G$. This fact is an immediate consequence of Thompson's normal p-complement theorem in J. Algebra 1 (1964), 43-46.

\section{REFERENCES}

1. R. Baer, Classes of finite groups and their properties, Illinois J. Math. 1 (1957), 115-187.

2. Z. Janko, Verallgemeinerung eines Satzes von B. Huppert und J. G. Thompson, Arch. Math. 12 (1961), 280-281.

3. W. R. Scott, Group theory, Prentice-Hall, Englewood Cliffs, N. J., 1964.

WEST VIRGINIA UNIVERSITY 